\title{
Hopf algebra of permutation pattern functions (Extended abstract)
}

\author{
Yannic Vargas $\left.\right|^{*}$ \\ Laboratoire de Combinatoire et d'Informatique Mathématique (LaCIM), UQAM, Canada
}

\begin{abstract}
We study permutation patterns from an algebraic combinatorics point of view. Using analogues of the classical shuffle and infiltration products for word, we define two new Hopf algebras of permutations related to the notion of permutation pattern. We show several remarkable properties of permutation patterns functions, as well their occurrence in other domains.

Résumé. On étudie les motifs de permutations d'un point de vue de la combinatoire algébrique. En utilisant des analogues des produits classiques de mélanges et d'infiltration, on définit deux nouvelles algèbres de Hopf de permutations reliées à la notion de motifs de permutations. On montre quelques identités remarquables des fonctions de motifs de permutations, ainsi que ses liens avec d'autres domaines.
\end{abstract}

Keywords: Pattern permutation, shuffle, infiltration, bialgebra, Hopf algebra, representative function

\section{Introduction}

Let $\mathbb{N}$ the set of nonnegative integers. For $n \in \mathbb{N}$, consider $\mathfrak{S}_{n}$ the set of permutations on $\{1,2, \ldots, n\}$. We denote $\lambda$ the empty permutation, thus $\mathfrak{S}_{0}=\{\lambda\}$. Also, let $\mathfrak{S}=\cup_{n \geq 0} \mathfrak{S}_{n}$ (disjoint union) the set of all permutations. Let $\mathbb{Q}^{\mathfrak{S}}$ be the set of all functions from $\mathfrak{S}$ to $\mathbb{Q}$. This is a commutative $\mathbb{Q}$-algebra when it is equipped by sum and the pointwise product: if $f, g \in \mathbb{Q}^{\mathfrak{S}}$ and $\sigma \in \mathfrak{S}$, then

$$
(f+g)(\sigma)=f(\sigma)+g(\sigma) \quad, \quad(f \cdot g)(\sigma):=f(\sigma) g(\sigma) .
$$

In this work we consider a special subalgebra of $\mathbb{Q}^{\mathfrak{S}}$ based on permutation patterns. If $w, \sigma \in \mathfrak{S}$, then $\sigma$ is said to be a pattern of $w$ if there exists a subsequence of entries of $w$ that has the same relative order as $\sigma$. We define $\left\{\begin{array}{c}w \\ \sigma\end{array}\right\}$ as the number of occurrences of $\sigma$ as a pattern of $w$. For example,

$$
\left\{\begin{array}{c}
25143 \\
132
\end{array}\right\}=|\{254,253,143\}|=3 .
$$

Notice that $\left\{\begin{array}{c}w \\ \lambda\end{array}\right\}=1$ and $\left\{\begin{array}{c}w \\ w\end{array}\right\}=1$, for all $w \in \mathfrak{S}$. Permutation patterns are considered in several domains, such as as theoretical computer science and enumerative combinatorics; namely, in problems like stacksorting (Knuth, $1968[8]$ ) and pattern avoidance. This work is intended to study permutation patterns from

*Email: vargas_lozada.yanniceuqam.ca. Partially supported by ISM and LaCIM 
an algebraic combinatorics point of view. The main theorem of this article (proved in section 5) is the following:

Theorem 1 The family of functions $w \mapsto\left\{\begin{array}{l}w \\ \sigma\end{array}\right\}$, for $\sigma \in \mathfrak{S}$, span a free commutative subalgebra of $\mathbb{Q}^{\mathfrak{S}}$.

To describe the algebraic structure in the above theorem, we introduce two new products in the vector space of permutations $\mathbb{Q S}$ : the super-shuffle $\underline{m}$, and the super-infiltration $\Uparrow$ (see sections 3.2 and 3.3, respectively). The first one interpolates between two products introduced by Malvenuto and Reutenauer in [10] (section 3, page 978). The super-infiltration is a natural generalization of the classical infiltration product for words (see [9], chapter 6.3, page 128). We also consider the following coproduct in $\mathbb{Q S}$ :

$$
\Delta_{\square}(\sigma)=\sum_{\sigma=\alpha \square \beta} \alpha \otimes \beta,
$$

where $\square$ is defined as follow: if $\alpha=a_{1} \cdots a_{n} \in \mathfrak{S}_{n}$ and $\beta=b_{1} \cdots b_{p} \in \mathfrak{S}_{p}$, then

$$
\alpha \square \beta:=a_{1} \cdots a_{n}\left(b_{1}+n\right) \cdots\left(b_{p}+n\right) \in \mathfrak{S}_{n+p} .
$$

Theorem 2 The $\mathbb{Q}$-vector space $\mathbb{Q S}$, endowed with the product of super-infiltration and the coproduct $\Delta_{\square}$, is a Hopf algebra. As an algebra, it is a free commutative $\mathbb{Q}$-algebra. The function

$$
\sigma \mapsto\left(w \mapsto\left\{\begin{array}{l}
w \\
\sigma
\end{array}\right\}\right)
$$

is an isomorphism from this algebra to the subalgebra of theorem 1.

Theorem (2) (proved in section 4) clearly implies Theorem (1). In particular, we have an analogue of a relation due to Chen, Fox and Lyndon (see [2]; also [9], page 131). This relation describes the algebraic structure of the subalgebras of theorems 1 and 2:

$$
\left\{\begin{array}{l}
w \\
\alpha
\end{array}\right\}\left\{\begin{array}{l}
w \\
\beta
\end{array}\right\}=\sum_{\sigma \in \mathfrak{S}}(\alpha \Uparrow \beta, \sigma)\left\{\begin{array}{l}
w \\
\sigma
\end{array}\right\} .
$$

We finish this work by showing several identities and properties of permutation pattern functions, as well some occurrence of this objects in other domains. These results are expanded on and proven in the manuscript [16] of the same name.

\section{Classical binomial coefficients of words, shuffle and infiltration}

In this section we present some basic notions on words and binomial coefficients of words. Most of the proofs of the results mentioned here can be found in [9]. The diligent reader will notice that these facts are straighforward to verify.

\subsection{Operations on words}

Let $\mathbb{N}$ be the set of non-negatives integers. For all $n \in \mathbb{N}$ we write $[n]:=\{1,2, \ldots, n\}$ and $[0]:=\emptyset$. An alphabet is a set $A$. The letter of the alphabet are the elements of $A$. A word over the alphabet $A$ is a finite sequence of elements of $A$. The set of all words over $A$ is denoted by $A^{*}$. The set $A^{*}$ is a monoid with the concatenation product and the empty word, denoted by $\lambda$, as neutral element for the concatenation. 
Let $w=a_{1} a_{2} \cdots a_{n}$ be a word in $A^{*}$. The length of $w$, denoted by $|w|$, is the number $n$ of letters of $w$. If $I=\left\{i_{1}<i_{2}<\cdots<i_{k}\right\}$ is a subset of $[n]$, let $w_{I}:=a_{i_{1}} a_{i_{2}} \cdots a_{i_{k}}$. A word $u \in A^{*}$ is said to be a subword of $w$ if there exist a subset $I$ of $[n]$ such that $u=w_{I}$. We write $u \subseteq w$ if $u$ is a subword of $w$. If $|w|=n$ and $I \subseteq[n]$ is fixed, we say that $w_{[n] \backslash I}$ is the complementary subword of $w_{I}$ in $w$.

The alphabet of a word $w \in A^{*}$ is the $\operatorname{set} \operatorname{alph}(w)$ of the letters ocurring in $w$. If the alphabet $A$ is totally ordered, and $u, v \in A^{*}$ are such that all letters in $v$ are greater than the letters in $u$, we write $\operatorname{alph}(u)<\operatorname{alph}(v)$.

\subsection{Formal series and non-commutative polynomials}

Let $A$ be a countable ordered alphabet and $\mathbb{K}$ a closed field. A formal series with coefficients in $\mathbb{K}$ and variables in $A$ is an infinite formal linear combination

$$
f=\sum_{w \in A^{*}}(f, w) w
$$

where $(f, w)$ is the value of $f$ on $w$. It is called the coefficient of $w$ in $f$. The set of all series is denoted by $\mathbb{K}\langle\langle A\rangle\rangle$. It acquires a $\mathbb{K}$-algebra structure, with

$$
(f+g, w)=(f, w)+(g, w) \quad \text { and } \quad(f g, w)=\sum_{w=u v}(f, u)(g, v) .
$$

The space $\mathbb{K}\langle\langle A\rangle\rangle$ is endowed with the following bilinear form: for all $f, g \in \mathbb{K}\langle\langle A\rangle\rangle$,

$$
\langle f, g\rangle:=\sum_{w \in A^{*}}(f, w)(g, w) .
$$

\subsection{Shuffle and infiltration products}

If $u \in A^{n}$ and $v \in A^{p}$, we define the shuffle product $w$ and the infiltration product $\uparrow$ of $u$ and $v$ as

$$
u ш v:=\sum_{\substack{I \uplus J=[n+p] \\ w_{I}=u, w_{J}=v}} w \quad, \quad u \uparrow v:=\sum_{\substack{I \cup J \subseteq[n+p] \\ w_{I}=u, w_{J}=v}} w .
$$

\section{Example 2.1}

$$
\begin{aligned}
b a ш a b & =b a a b+b a a b+b a b a+a b a b+a b b a+a b b a \\
& =2 a b b a+a b a b+2 b a a b+b a b a . \\
b a \uparrow a b & =b a b+a b a+b a a b+b a a b+b a b a+a b a b+a b b a+a b b a \\
& =b a b+a b a+b a w a b \\
& =a b a+b a b+2 a b b a+a b a b+2 b a a b+b a b a .
\end{aligned}
$$

We remark that the infiltration product contains the shuffle product. The shuffle product was introduced by Eilenberg and MacLane in [3]. Chen, Fox and Lyndon introduced in [2] (Theorem 3.9, page 93) the infiltration product in an algebraic framework; Ochsenschläger (see [12]) defined this product independently in the context of binomial coefficients of words (see the next section). Both products are associative and commutative (see [9], proposition 6.3.12 and proposition 6.3.15). 
The shuffle and infiltration product are related by the following identity ([9], Theorem. 6.3.18.):

$$
\left(u ш \mathbf{A}^{*}\right) \times\left(v ш \mathbf{A}^{*}\right)=(u \uparrow v) ш \mathbf{A}^{*},
$$

where $\mathbf{A}^{*}$ is the formal series $\mathbf{A}^{*}:=\sum_{w \in A^{*}} w$ and $\times$ denotes the Hadamard product. For $f, g \in$ $\mathbb{K}\langle\langle A\rangle\rangle$ and $w \in A^{*}$, it is defined by $(f \times g, w):=(f, w)(g, w)$.

Both products can also be defined from a general recursive formula. Let $\bar{A}:=A^{*} \cup\{0\}$, where 0 is the zero of $\mathbb{K}$. A quasi-shuffle product $\bullet$ in $\mathbb{K}\langle A\rangle$ is a bilinear product such that $\lambda \bullet w=w \bullet \lambda=w$ for any word $w$, and, for any $\mathrm{a}, \mathrm{b} \in A$ and $u, v \in A^{*}$,

$$
\mathrm{a} u \bullet \mathrm{b} v=\mathrm{a}(u \bullet \mathrm{b} v)+\mathrm{b}(\mathrm{a} u \bullet v)+[\mathrm{a}, \mathrm{b}](u \bullet v),
$$

where $[-,-]: \bar{A} \times \bar{A} \rightarrow \bar{A}$ is a function satisfying:

(S1) $[\mathrm{a}, 0]=0$, for all $\mathrm{a} \in \bar{A}$;

(S2) $[\mathrm{a}, \mathrm{b}]=[\mathrm{b}, \mathrm{a}]$, for all $\mathrm{a}, \mathrm{b} \in \bar{A}$;

(S3) $[[\mathrm{a}, \mathrm{b}], \mathrm{c}]=[\mathrm{a},[\mathrm{b}, \mathrm{c}]]$, for all $\mathrm{a}, \mathrm{b}, \mathrm{c} \in \bar{A}$;

This construction is old and has been rediscovered many times. As mentioned by one the referees, it probably appeared first in a work of Newman and Radford from 1979 [11]. In the last decade, it appeared in work of Hazewinkel and (in a particular case) in work of Hoffman. Enjalbert and Minh consider the definition above ([4], definition 2.1). Hoffman defined this product with an additional graded property on the words in $A^{*}$ (see [6], page 51). When [a, b] $:=0$ for all letters in $A^{*} \backslash\{\lambda\}$, we obtain the shuffle product; if $[\mathrm{a}, \mathrm{b}]:=\delta_{\mathrm{a}, \mathrm{b}} \mathrm{a}$, we obtain the infiltration product.

Consider now the deconcatenation coproduct:

$$
\Delta(w)=\sum_{w=u v} u \otimes v .
$$

Proposition $2.2(\mathbb{K}\langle A\rangle, \bullet, \Delta)$ is a bialgebra.

See [4], proposition 2.3, for a proof and [6], theorem 3.1, for the graded case.

\subsection{Binomial coefficients of words}

An important tool for counting subwords is the notion of binomial coefficient of words, a generalization of the classical binomial coefficient for integers. Given $u, v \in A^{*}$, the number of subwords of $u$ that are equal to $v$ is called the binomial coefficient of $u$ by $v$ and is denoted by $\left(\begin{array}{l}u \\ v\end{array}\right)$. For example,

$$
\left(\begin{array}{c}
\text { bloab } \\
\text { bab }
\end{array}\right)=2 \text {. }
$$

If $m, n \in \mathbb{N}$ are such that $n \leq m$ and a is a letter in $A$, we remark that

$$
\left(\begin{array}{l}
\mathrm{a}^{m} \\
\mathrm{a}^{n}
\end{array}\right)=\left(\begin{array}{l}
m \\
n
\end{array}\right),
$$

where the right hand side denotes the classical binomial coefficient. of integers. 
The binomial coefficients of words are closely related to the shuffle and infiltration products. For instance, the coefficient of $u$ in the infiltration product of $u$ and $v$ is

$$
(u \uparrow v, u)=\left|\left\{(I, J): I \cup J \subseteq[|u|+|v|], u_{I}=u, u_{J}=v\right\}\right|=\left|\left\{J \subseteq[|u|]: u_{J}=v\right\}\right|=\left(\begin{array}{l}
u \\
v
\end{array}\right) .
$$

A way to encode all subwords of a fixed word is given by the Magnus transformation: it is the algebra endomorphism $\mu$ of $\mathbb{Z}\langle A\rangle$ defined by $\mu(\mathrm{a})=\lambda+\mathrm{a}$, for all $\mathrm{a} \in A$. For example,

$$
\begin{aligned}
\mu(\mathrm{bbab}) & =(\lambda+\mathrm{b})(\lambda+\mathrm{b})(\lambda+\mathrm{a})(\lambda+\mathrm{b}) \\
& =\lambda+\mathrm{a}+3 \mathrm{~b}+\mathrm{ab}+2 \mathrm{ba}+3 \mathrm{bb}+2 \mathrm{bab}+\mathrm{bba}+\mathrm{b} b \mathrm{~b}+\mathrm{bbab} .
\end{aligned}
$$

Is easy to see that

$$
\mu(u)=\sum_{v \in A^{*}}\left(\begin{array}{l}
u \\
v
\end{array}\right) v,
$$

for all $u \in A^{*}$. The Magnus transformation is an automorphism ([9], cor. 6.3.8.). The inverse is given by

$$
\mu^{-1}(u)=\sum_{v \in A^{*}}(-1)^{|u|+|v|}\left(\begin{array}{l}
u \\
v
\end{array}\right) v .
$$

It can be shown (by induction on the length) that the shuffle of a word $v \in A^{*}$ with the formal series $\mathbf{A}^{*}$ is:

$$
v ш \mathbf{A}^{*}=\sum_{u \in A^{*}}\left(\begin{array}{l}
u \\
v
\end{array}\right) u
$$

(see [9], prop. 6.3.13.). This implies that shuffling with $\mathbf{A}^{*}$ is the adjoint operator of the Magnus transformation:

$$
\langle\mu(u), v\rangle=\left(\begin{array}{l}
u \\
v
\end{array}\right)=\left\langle v w \mathbf{A}^{*}, u\right\rangle .
$$

From the series relation (3) involving shuffle and infiltration, we obtain

$$
\left\langle u ш \mathbf{A}^{*}, w\right\rangle\left\langle v w \mathbf{A}^{*}, w\right\rangle=\left\langle(u \uparrow v) w \mathbf{A}^{*}, w\right\rangle,
$$

for all $u, v, w \in A^{*}$. By (9), this is equivalent to

$$
\langle u, \mu(w)\rangle\langle v, \mu(w)\rangle=\langle u \uparrow v, \mu(w)\rangle .
$$

This implies the following "product rule" between binomial coefficients of words

$$
\left(\begin{array}{l}
w \\
u
\end{array}\right)\left(\begin{array}{l}
w \\
v
\end{array}\right)=\sum_{s \in A^{*}}(u \uparrow v, s)\left(\begin{array}{l}
w \\
s
\end{array}\right) .
$$

Formally, the equation above means that the span of the set of functions $\left\{w \mapsto\left(\begin{array}{l}w \\ u\end{array}\right): u \in A^{*}\right\}$ is a subalgebra of the algebra of functions from $A^{*}$ to $\mathbb{Q}$, where the ring structure is defined pointwise. In the next sections we develop an analogue of this result for permutation pattern functions. 


\section{Permutation pattern, supershuffle and superinfiltration}

\subsection{Operations on permutations}

Let $n \in \mathbb{N}$. A permutation on $[n]$ is a bijection from $[n]$ to $[n]$. Consider $\mathfrak{S}_{n}$ the set of permutations on $[n]$. We denote $\lambda$ the empty permutation, thus $\mathfrak{S}_{0}=\{\lambda\}$. Also, let $\mathfrak{S}=\cup_{n \geq 0} \mathfrak{S}_{n}$ (disjoint union) the set of all permutations. Let $A$ be a total ordered alphabet. The standard permutation of a word $w=a_{1} \cdots a_{n}$ on $A^{*}$ is the unique permutation, denoted $\operatorname{st}(w)$, such that for any $i, j \in[n]$,

$$
\operatorname{st}(w)_{i}<\operatorname{st}(w)_{j} \Longleftrightarrow a_{i}<a_{j} \text { or }\left(a_{i}=a_{j} \text { and } i<j\right) .
$$

The permutation st $(w)$ may be obtained by numbering from left to right the letters of $w$, starting with the smallest letter, then the second smallest letter, and so on. For example, if $\mathrm{a}<\mathrm{b}<\mathrm{c}$ we have

$$
\text { If } \sigma \in \mathfrak{S}_{n} \text { and } k \in \mathbb{N} \text { let } \quad \begin{array}{c|c}
u & \text { c b b a b b a c b } \\
\hline \text { st }(u) & 834156297 \\
{[\sigma]_{k}:=\left(\sigma_{1}+k\right)\left(\sigma_{2}+k\right) \cdots\left(\sigma_{n}+k\right) .}
\end{array}
$$

The set of permutations $\mathfrak{S}$ is an associative monoid with the product

$$
\alpha \square \beta:=\alpha[\beta]_{n}=\alpha_{1} \cdots \alpha_{n}\left(\beta_{1}+n\right) \cdots\left(\beta_{p}+n\right) \in \mathfrak{S}_{n+p},
$$

if $\alpha=\alpha_{1} \cdots \alpha_{n} \in \mathfrak{S}_{n}$ and $\beta=\beta_{1} \cdots \beta_{p} \in \mathfrak{S}_{p}$. A permutation $\sigma$ is said indecomposable if $\sigma=\alpha \square \beta$ implies $\alpha=\lambda$ or $\beta=\lambda$. Let Ind the set of the indecomposable permutations. Then, $\mathfrak{S}$ is a free monoid generated by Ind.

\subsection{Permutation pattern}

If $w, \sigma \in \mathfrak{S}$, then $\sigma$ is said to be a pattern of $w$ if there exists a subsequence of entries of $w$ that has the same relative order as $\sigma$. We define $\left\{\begin{array}{c}w \\ \sigma\end{array}\right\}$ as the number of permutation patterns of $\sigma$ in $w$. In terms of standardization, this number can be described as

$$
\left\{\begin{array}{l}
w \\
\sigma
\end{array}\right\}=|\{u \subseteq w: \operatorname{st}(u)=\sigma\}| .
$$

Notice that $\left\{\begin{array}{c}w \\ \lambda\end{array}\right\}=1$ and $\left\{\begin{array}{c}w \\ w\end{array}\right\}=1$, for all $w \in \mathfrak{S}$.

\section{Example 3.1}

$$
\left\{\begin{array}{c}
25143 \\
312
\end{array}\right\}=|\{514,513\}|=2 .
$$

Some combinatorial numbers can be deduced from the number of permutation patterns of a permutation. For instance, if $k \leq n$, we have

Also, if $\sigma \in \mathfrak{S}$, then

$$
\left\{\begin{array}{l}
12 \cdots n \\
12 \cdots k
\end{array}\right\}=\left(\begin{array}{l}
n \\
k
\end{array}\right) \text {. }
$$

$$
\left\{\begin{array}{c}
\sigma \\
12
\end{array}\right\}=\operatorname{coinv}(\sigma) \text { and }\left\{\begin{array}{c}
\sigma \\
21
\end{array}\right\}=\operatorname{inv}(\sigma)
$$

where $\operatorname{coinv}(\sigma)$ and $\operatorname{inv}(\sigma)$ are the number of coinversions and inversions of $\sigma$, respectively:

$$
\operatorname{coinv}(\sigma):=\mid\left\{(i, j): i<j \text { and } \sigma_{i}<\sigma_{j}\right\}|\quad, \quad \operatorname{inv}(\sigma):=|\left\{(i, j): i<j \text { and } \sigma_{i}>\sigma_{j}\right\} \mid .
$$




\subsection{Supershuffle}

In [10], Malvenuto and Reutenauer introduced two products in $\mathbb{K} \mathfrak{S}$. If $\alpha \in \mathfrak{S}_{n}$ and $\beta \in \mathfrak{S}_{p}$, let

$$
\alpha * \beta:=\sum_{\begin{array}{c}
w=u v \\
\operatorname{st}(u)=\alpha, \operatorname{st}(v)=\beta \\
\operatorname{alph}(u) \uplus \operatorname{alph}(v)=[n+p]
\end{array}} w \quad \text { and } \quad \alpha *^{\prime} \beta:=\alpha w[\beta]_{n}=\sum_{\begin{array}{c}
I \uplus J=[n+p] \\
w_{I}=\alpha, w_{J}=[\beta]_{n} \\
\operatorname{alph}\left(w_{I}\right) \uplus \operatorname{alph}\left(w_{J}\right)=[n+p]
\end{array}} w
$$

It is proved that $(\mathbb{K S}, *)$ and $\left(\mathbb{K S}, *^{\prime}\right)$ are isomorphic algebras. The isomorphism is given by $\theta$ : $\mathbb{K} \mathfrak{S} \rightarrow \mathbb{K} \mathfrak{S}$, where $\theta$ is defined on permutations by $\theta(\sigma)=\sigma^{-1}$. It is possible to rewrite these two products using the notion of binomial coefficients of words: if $u, v, w$ are words on an alphabet $A$, let $\left(\begin{array}{c}w \\ u, v\end{array}\right)$ be the number of complementary subwords $\left(w_{1}, w_{2}\right)$ of $w$ such that $w_{1}=u$ and $w_{2}=v$. Using the isomorphism $\theta$, we have

$$
\alpha * \beta=\sum_{w \in \mathfrak{S}_{n+p}}\left(\begin{array}{c}
w \\
\alpha^{-1},\left[\beta^{-1}\right]_{n}
\end{array}\right) w^{-1} \quad \text { and } \quad \alpha *^{\prime} \beta=\sum_{w \in \mathfrak{S}_{n+p}}\left(\begin{array}{c}
w \\
\alpha,[\beta]_{n}
\end{array}\right) w
$$

We define a new product in $\mathbb{K} \mathfrak{S}$ which interpolates between $*$ and $*^{\prime}$. If $\alpha \in \mathfrak{S}_{n}$ and $\beta \in \mathfrak{S}_{p}$, the supershuffle of $\alpha$ and $\beta$ is

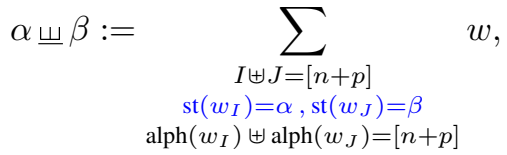

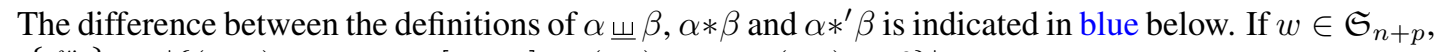
let $\left\{\begin{array}{c}w \\ \alpha, \beta\end{array}\right\}:=\left|\left\{(I, J): I \uplus J=[n+p], \operatorname{st}\left(w_{I}\right)=\alpha, \operatorname{st}\left(w_{J}\right)=\beta\right\}\right|$. Then, by definition of $\underline{w}$ we have

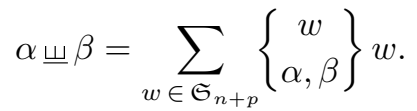

Observe that $\left\{\begin{array}{c}w \\ \alpha, \beta\end{array}\right\}=\left\{\begin{array}{c}w \\ \beta, \alpha\end{array}\right\}$. So, the product $\underline{m}$ is commutative. Also, we remark that $\left\{\begin{array}{c}w \\ \alpha, \lambda\end{array}\right\}=\left\{\begin{array}{c}w \\ \alpha\end{array}\right\}$, for all $w, \alpha \in \mathfrak{S}$. It is straightforward to prove that $\underline{m}$ is associative (see [16] for more details).

\section{Example 3.2}

$$
\begin{aligned}
21 щ 1 & =213+132+2 \cdot(312+231)+3 \cdot 321 . \\
21 щ 12 & =1243+3142+4231+1324+2413+2134+4312+3421 \\
& +2 \cdot(4132+2314+1423+3124+4213+1342+2431) \\
& +3 \cdot(2341+3214+1432+4123) .
\end{aligned}
$$

We have, for example, $(21 \underline{\underline{1}} 12,2341)=\left\{\begin{array}{c}2341 \\ 21,12\end{array}\right\}=|\{(21,34),(31,24),(41,23)\}|=3$. 


\subsection{Superinfiltration}

We present now an analogue of the infiltration product for permutations. Let $\alpha \in \mathfrak{S}_{n}, \beta \in \mathfrak{S}_{p}$. The superinfiltration of $\alpha$ and $\beta$ is

$$
\alpha \Uparrow \beta:=\sum_{\substack{I \cup J \subseteq[n+p] \\|I|=n,|J|=p \\ \operatorname{st}\left(w_{I}\right)=\alpha, \operatorname{st}\left(w_{J}\right)=\beta}} w .
$$

Let $\left[\begin{array}{c}w \\ \alpha, \beta\end{array}\right]:=(\alpha \Uparrow \beta, w)$. The superinfiltration contains the supershuffle, as the infiltration contains the shuffle product of words. Indeed:

$$
\left[\begin{array}{c}
w \\
\alpha, \beta
\end{array}\right]=\left|\left\{(u, v): \begin{array}{c}
u \subseteq w, v \subseteq w \\
\operatorname{st}(u)=\alpha, \operatorname{st}(v)=\beta
\end{array}\right\}\right|
$$

and

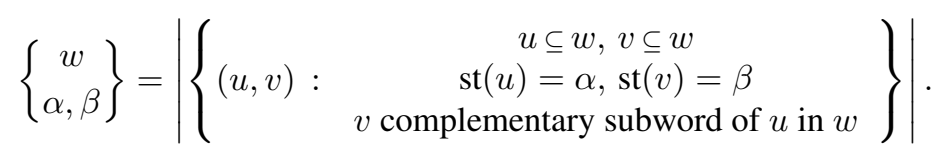

From (20), it is clear that the superinfiltration is commutative. Also, it is straightforward to prove that $\Uparrow$ is associative (see [16] for more details). As for the supershuffle product, we remark that $\left[\begin{array}{c}w \\ \alpha, \lambda\end{array}\right]=\left\{\begin{array}{c}w \\ \alpha\end{array}\right\}$.

Example 3.3 We have:

$$
\begin{aligned}
& {\left[\begin{array}{c}
2341 \\
231,21
\end{array}\right]=\mid\{(231,21),(231,31),(231,41),(241,21),} \\
& (241,31),(241,41),(341,21)(341,31),(341,41)\} \mid=9 . \\
& {\left[\begin{array}{c}
231 \\
231,21
\end{array}\right]=|\{(231,21),(231,31)\}|=2 .}
\end{aligned}
$$

Then:

$$
231 \Uparrow 21=231 \underline{\Perp} 21+\cdots+9 \cdot 2341+\cdots+2 \cdot 231 .
$$

The map $\theta$ behaves well with the supershuffle and the superinfiltration product.

Proposition 3.4 Let $w, \alpha, \beta \in \mathfrak{S}$. Then $\left\{\begin{array}{c}w \\ \alpha, \beta\end{array}\right\}=\left\{\begin{array}{c}w^{-1} \\ \alpha^{-1}, \beta^{-1}\end{array}\right\}$ and $\left[\begin{array}{c}w \\ \alpha, \beta\end{array}\right]=\left[\begin{array}{c}w^{-1} \\ \alpha^{-1}, \beta^{-1}\end{array}\right]$. In particular,

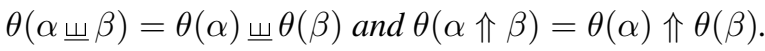

\section{Hopf algebras of supershuffle and superinfiltration}

For basic definitions and facts about bialgebras and Hopf algebras see [5], [7] or [15]. The supershuffle and superinfiltration products satisfy the following property:

Lemma 4.1 Let $\sigma, \pi, \alpha, \beta \in \mathfrak{S}$. Then

$$
\left\{\begin{array}{c}
\sigma \square \pi \\
\alpha, \beta
\end{array}\right\}=\sum_{\substack{\alpha=\alpha^{\prime} \square \alpha^{\prime \prime} \\
\beta=\beta^{\prime} \square \beta^{\prime \prime}}}\left\{\begin{array}{c}
\sigma \\
\alpha^{\prime}, \beta^{\prime}
\end{array}\right\}\left\{\begin{array}{c}
\pi \\
\alpha^{\prime \prime}, \beta^{\prime \prime}
\end{array}\right\} \quad \text { and } \quad\left[\begin{array}{c}
\sigma \square \pi \\
\alpha, \beta
\end{array}\right]=\sum_{\substack{\alpha=\alpha^{\prime} \square \alpha^{\prime \prime} \\
\beta=\beta^{\prime} \square \beta^{\prime \prime}}}\left[\begin{array}{c}
\sigma \\
\alpha^{\prime}, \beta^{\prime}
\end{array}\right]\left[\begin{array}{c}
\pi \\
\alpha^{\prime \prime}, \beta^{\prime \prime}
\end{array}\right] .
$$


These identities can be seen in an algebraic framework (i) let $\mu_{\underline{m}}$ and $\mu_{\Uparrow}$ be the bilinear extensions of the supershuffle and superinfiltration products, respectively, and consider the map $\Delta_{\square}: \mathbb{K} \mathfrak{S} \rightarrow \mathbb{K} \mathfrak{S} \otimes \mathbb{K} \mathfrak{S}$ defined by

$$
\Delta_{\square}(\sigma):=\sum_{\sigma=\alpha \square \beta} \alpha \otimes \beta .
$$

Then, the lemma above said that $\Delta_{\square}$ is a homomorphism for both products $\mu_{\underline{\varpi}}$ and $\mu_{\Uparrow}$. In analogy with proposition 2.2 , we have:

Theorem $4.2\left(\mathbb{K} \mathfrak{S}, \mu_{\underline{\Perp}}, \Delta_{\square}\right)$ and $\left(\mathbb{K S}, \mu_{\Uparrow}, \Delta_{\square}\right)$ are bialgebras.

By a theorem of Milnor and Moore, every graded and locally finite bialgebra possesses an antipode, and then it is a Hopf algebra (for more details see for example [1], page 18, section 5). Since $\left(\mathbb{K S}, \mu_{\varpi}, \Delta_{\square}\right)$ satisfies these hypothesis, we have

Theorem $4.3\left(\mathbb{K S}, \mu_{\varpi}, \Delta_{\square}\right)$ is a Hopf algebra.

The algebra $\left(\mathbb{K S}, \mu_{\Uparrow}\right)$ is not graded by the length (see the example (3.3) ) and then an antipode does not come "for free" from the the theorem of Milnor-Moore. However, the bialgebra $\left(\mathbb{K S}, \mu_{\Uparrow}, \Delta_{\square}\right)$ indeed possesses a structure of Hopf algebra. The description of the antipodes of $\left(\mathbb{K} \mathfrak{S}, \mu_{\underline{\underline{m}}}, \Delta_{\square}\right)$ and $\left(\mathbb{K S}, \mu_{\Uparrow}, \Delta_{\square}\right)$ is discussed in the section (4.2).

The application $\Delta_{\square}$ is a variant of the coproduct in the Malvenuto-Reutenauer Hopf algebra, with respect to its "monomial functions" introduced by Aguiar and Sottile [1]. We remark that $\Delta_{\square}$ is not cocommutative.

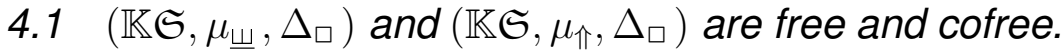

Let $A$ a totally ordered alphabet and $<_{\operatorname{lex}}$ the lexicographic order on $A^{*}$. Recall that a Lyndon word over $A$ is a non-empty word which is strictly smaller than each of its proper right factors, for $<_{\text {lex }}$. We denote the set of all Lyndon words on $A$ by $\mathcal{L}$. Any word $w \in A^{*}$ can be factorized in a unique way as a decreasing product (for lexicographic order) of Lyndon words (see [2], [9] or [15]). In other words, $w=\ell_{1}^{r_{1}} \cdots \ell_{k}^{r_{k}}$, where $\ell_{i} \in \mathcal{L}$ and $\ell_{i}>_{\operatorname{lex}} \ell_{j}$ if $i<j$. We will use the following technical result:

Lemma 4.4 (Radford[14]) Let $w=\ell_{1}^{r_{1}} \cdots \ell_{k}^{r_{k}} \in A^{*}$, with $\ell_{1}, \ldots, \ell_{k} \in \mathcal{L}$. The polynomial

$$
Q_{w}=1 /\left(r_{1} ! \cdots r_{k} !\right) \ell_{1}^{ш r_{1}} ш \cdots ш \ell_{k}^{ш r_{k}}
$$

can be written as $w+R_{w}$, where $R_{w}$ contains only words of weight equal to the weight of $w$, but smaller than $w$ (for the lexicographic order).

In order to prove Theorem 2, we will need to introduce analogous notions for permutations. First, consider the following total order on the set of indecomposable permutations Ind: if $\alpha, \beta \in$ Ind, we define

$$
\alpha<\beta \quad \text { if } \quad|\alpha|<|\beta|, \quad \text { or } \quad|\alpha|=|\beta| \quad \text { and } \quad \alpha<_{\operatorname{lex}} \beta \text {. }
$$

Then, the elements in Ind are totally ordered as $1<21<231<312<321<2341<\cdots$. We define a Lyndon permutation as a permutation $\sigma$ such that $\sigma \neq \lambda$, and for each factorization $\sigma=\alpha \square \beta$, with $\alpha, \beta \neq \lambda$, one has $\sigma<\beta$. We denote the set of all Lyndon permutations by $\mathcal{L}_{\mathfrak{S}}$.

\footnotetext{
(i) These identities are also related with the notion of section coefficients of a bialgebra, as in [7]. We discuss this notion related to permutation pattern function in a future work.
} 
Lemma 4.5 Any permutation $\sigma \in \operatorname{Ind}^{*} \simeq \mathfrak{S}$ can be factorized in a unique way as a decreasing product (for the order $<$ ) of Lyndon permutations.

Consider now the following variation of the shuffle product: let $ш \square$ be the relative shuffle to the alphabet Ind. Formally, if $\sigma, \pi \in$ Ind $^{*} \simeq \mathfrak{S}$ and $\alpha, \beta \in$ Ind,

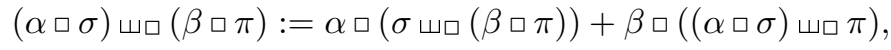

and $\sigma ш_{\square} \lambda=\lambda ш_{\square} \sigma=\sigma$. For instance,

$(21 \square 1)$ ш $21=21 \square 1 \square 21+21 \square 21 \square 1+21 \square 21 \square 1=21 \square 1 \square 21+2 \cdot(21 \square 21 \square 1)=21354+2 \cdot(21435)$.

For each $\sigma \in \mathfrak{S}$, let $|\sigma|_{\square}$ be the relative length of $\sigma$ over Ind; it is the number of letters of $\sigma$ as a word in the alphabet Ind. For example, $|231465|_{\square}=3$, because $231465=231 \square 1 \square 21$.

Lemma 4.6 Let $\alpha \in \mathfrak{S}_{n}, \beta \in \mathfrak{S}_{p}$. Then

$$
\alpha \underline{\Perp}=\alpha ш \square \beta+\text { sum of permutations } \pi \in \mathfrak{S}_{n+p} \text { such that }|\pi|_{\square}<|\alpha|_{\square}+|\beta|_{\square} \text {. }
$$

Proof of theorem (2): Let $\ell_{1}, \ldots, \ell_{k} \in \mathcal{L}_{\mathfrak{S}}$, with $\ell_{i} \in \mathfrak{S}_{n_{i}}$ and $\left|\ell_{i}\right|_{\square}=s_{i}$. Consider $\sigma=\ell_{1}^{\square r_{1}} \square \cdots \square \ell_{k}^{\square r_{k}} \in$ $\mathfrak{S}_{n}$, for some $n \in \mathbb{N}$. By the lemmas (4.4) and (4.6) we have

$$
\begin{aligned}
& \ell_{1}^{\underline{\varpi} r_{1}} \underline{\varpi} \cdots \ell_{k}^{\underline{\varpi} r_{k}}=r_{1} ! \cdots r_{k} !\left(\sigma+\text { sum of permutations } \pi^{\prime \prime} \in \mathfrak{S}_{n} \text { such that } \pi^{\prime \prime}<\sigma\right) \\
& + \text { sum of permutations } \pi^{\prime} \in \mathfrak{S}_{n} \text { such that }\left|\pi^{\prime}\right|_{\square}<r_{1} s_{1}+\cdots+r_{k} s_{k}
\end{aligned}
$$

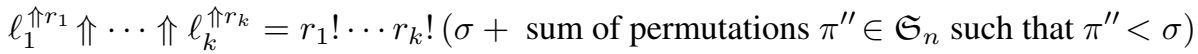

$$
\begin{aligned}
& + \text { sum of permutations } \pi^{\prime} \in \mathfrak{S}_{n} \text { such that }\left|\pi^{\prime}\right|_{\square}<r_{1} s_{1}+\cdots+r_{k} s_{k} \\
& + \text { sum of permutations } \pi \in \mathfrak{S}_{p}, p<n \text {. }
\end{aligned}
$$

By triangularity, $(\mathbb{K S}, \underline{\mathcal{S}})$ and $(\mathbb{K} \mathfrak{S}, \Uparrow)$ are freely generated by the elements in $\mathcal{L}_{\mathfrak{S}}$. Also, by the dual version of the theorem 6.1 in [13], $\left(\mathbb{K} \mathfrak{S}, \Delta_{\square}\right)$ is cofreely generated by the elements in Ind. Then, $\left(\mathbb{K S}, \mu_{\underline{\varpi}}, \Delta_{\square}\right)$ and $\left(\mathbb{K} \mathfrak{S}, \mu_{\Uparrow}, \Delta_{\square}\right)$ are free and cofree bialgebras.

\subsection{The antipodes of $\left(\mathbb{K} \mathfrak{S}, \mu_{\underline{\varpi}}, \Delta_{\square}\right)$ and $\left(\mathbb{K} \mathfrak{S}, \mu_{\Uparrow}, \Delta_{\square}\right)$}

We give now a description of the antipode for $\left(\mathbb{K} \mathfrak{S}, \mu_{\underline{\varpi}}, \Delta_{\square}\right)$ and $\left(\mathbb{K} \mathfrak{S}, \mu_{\Uparrow}, \Delta_{\square}\right)$. The proof follows easily by induction on the relative length of a permutation.

Proposition 4.7 Let $\sigma=\sigma_{1} \square \sigma_{2} \square \cdots \square \sigma_{n}$ a permutation such that $|\sigma|_{\square}=n$. Then $\left(\mathbb{K S}, \mu_{\varpi}, \Delta_{\square}\right)$ and $\left(\mathbb{K} \mathfrak{S}, \mu_{\Uparrow}, \Delta_{\square}\right)$ possess antipodes $S_{\underline{\Perp}}$ and $S_{\Uparrow}$, respectively, given by

$$
\begin{gathered}
S_{\underline{\uplus}}(\sigma)=\sum_{\left(i_{1}, \ldots, i_{k}\right) \in \operatorname{Comp}_{n}}(-1)^{k}\left(\sigma_{1} \square \cdots \square \sigma_{i_{1}}\right) \underline{m}\left(\sigma_{i_{1}+1} \square \cdots \square \sigma_{i_{1}+i_{2}}\right) \underline{m} \underline{w}\left(\sigma_{i_{1}+\cdots+i_{k-1}+1} \square \cdots \square \sigma_{n}\right), \\
S_{\Uparrow}(\sigma)=\sum_{\left(i_{1}, \ldots, i_{k}\right) \in \operatorname{Comp}_{n}}(-1)^{k}\left(\sigma_{1} \square \cdots \square \sigma_{i_{1}}\right) \Uparrow\left(\sigma_{i_{1}+1} \square \cdots \square \sigma_{i_{1}+i_{2}}\right) \Uparrow \cdots \Uparrow\left(\sigma_{i_{1}+\cdots+i_{k-1}+1} \square \cdots \square \sigma_{n}\right),
\end{gathered}
$$

where Comp ${ }_{n}$ is the set of compositions of $n$.

Corollary $4.8\left(\mathbb{K S}, \mu_{\Uparrow}, \Delta_{\square}\right)$ is a Hopf algebra. 


\section{Hopf algebra of permutation pattern functions}

For each $\sigma \in \mathfrak{S}$, let $\varphi_{\sigma}: \mathfrak{S} \rightarrow \mathbb{Q}$ be defined by

$$
\varphi_{\sigma}(w):=\left\{\begin{array}{l}
w \\
\sigma
\end{array}\right\}
$$

for all $w \in \mathfrak{S}$. We call $\varphi_{\sigma}$ the permutation pattern function related to $\sigma$. It is straightforward to verify that the collection $\left\{\varphi_{\sigma}: \sigma \in \mathfrak{S}\right\}$ is linearly independent in $\mathbb{Q}^{\mathfrak{S}}$.

Proposition 5.1 Let $\alpha, \beta \in \mathfrak{S}$. The pointwise product $\varphi_{\sigma} \cdot \varphi_{\beta}$ in $\mathbb{Q}^{\mathfrak{S}}$ is given by

$$
\varphi_{\alpha} \cdot \varphi_{\beta}=\sum_{\sigma \in \mathfrak{S}}\left[\begin{array}{c}
\sigma \\
\alpha, \beta
\end{array}\right] \varphi_{\sigma} .
$$

This implies the formula (1). Let $\mathcal{P} \mathcal{P} \mathcal{F}=\mathbb{Q}\left\{\varphi_{\sigma}: \sigma \in \mathfrak{S}\right\}$. We equip the vector space $\mathcal{P} \mathcal{P} \mathcal{F}$ with the coproduct

$$
\Delta\left(\varphi_{\sigma}\right):=\sum_{\sigma=\alpha \square \beta} \varphi_{\alpha} \otimes \varphi_{\beta} .
$$

Theorem 5.2 The vector space $\mathcal{P} \mathcal{P} \mathcal{F}$, with the pointwise product and the coproduct $\Delta$, is a free and cofree Hopf algebra, isomorphic to $\left(\mathbb{Q S}, \mu_{\Uparrow}, \Delta_{\square}\right)$ by the map $\sigma \mapsto \varphi_{\sigma}$. It is a subalgebra of $\mathbb{Q}^{\mathfrak{S}}$, freely generated by the set $\left\{\varphi_{\sigma}: \sigma \in \mathcal{L}_{\mathfrak{S}}\right\}$.

A characterization of the permutation pattern functions The relation 28 permit to describe the permutation pattern functions, as follow:

Theorem 3 Let $s: \mathfrak{S} \rightarrow \mathbb{N}$ be a nonzero function such that, for every $\alpha, \beta \in \mathfrak{S}$,

Then, there exists $\sigma \in \mathfrak{S}$ such that $s=\varphi_{\sigma}$.

$$
s(\alpha) s(\beta)=\sum_{\sigma \in \mathfrak{S}}\left[\begin{array}{c}
\sigma \\
\alpha, \beta
\end{array}\right] s(\sigma) .
$$

Magnus transformation for permutation patterns. Several identities for the binomial coefficients of words can be transfered to the framework of permutation pattern functions. We have the following analogue of the Magnus transformation (compare with (6) and (7)): let $M:=\sum_{\sigma \in \mathfrak{S}} \varphi_{\sigma} \sigma$. Then:

Theorem 4 In the monoid-algebra $\mathbb{Z}[\mathfrak{S}]$ of the free monoid (S, $\square)$, the function $w \mapsto M(w)$ define an automorphism. The inverse of this map is given by

$$
M^{-1}(w)=\sum_{\sigma \in \mathfrak{S}}(-1)^{|w|+|\sigma|}\left\{\begin{array}{l}
w \\
\sigma
\end{array}\right\} \sigma .
$$

Permutation pattern functions as representative functions The space $\mathbb{Q}^{\mathfrak{S}}$ is a $(\mathfrak{S}, \square)$-module via $(f \cdot \sigma)(\pi):=f(\sigma \square \pi)$, for all $f \in \mathbb{Q}^{\mathfrak{S}}$ and $\sigma, \pi \in \mathfrak{S}$. Recall that a function $f \in \mathbb{Q}^{\mathfrak{S}}$ is representative if the set $\{f \cdot \sigma\}_{\sigma \in \mathfrak{S}}$ span a finite dimensional $\mathbb{Q}$-vector space. Let $\alpha, \sigma \in \mathfrak{S}$. If we put $\beta=\lambda$ in the first formula of 4.1), we obtain

$$
\left(\varphi_{\alpha} \cdot \sigma\right)(\pi)=\varphi_{\alpha}(\sigma \square \pi)=\left\{\begin{array}{c}
\sigma \square \pi \\
\alpha
\end{array}\right\}=\sum_{\alpha=\alpha^{\prime} \square \alpha^{\prime \prime}}\left\{\begin{array}{c}
\sigma \\
\alpha^{\prime}
\end{array}\right\}\left\{\begin{array}{c}
\pi \\
\alpha^{\prime \prime}
\end{array}\right\}=\sum_{\alpha=\alpha^{\prime} \square \alpha^{\prime \prime}}\left\{\begin{array}{c}
\sigma \\
\alpha^{\prime}
\end{array}\right\} \varphi_{\alpha^{\prime \prime}}(\pi) .
$$

Theorem 5 For each $\alpha \in \mathfrak{S}, \varphi_{\alpha}$ is a representative function.

This result is a generalization of Lemma 6.6 - (iii) of [15], where it is proved that subword functions (functions of the form $w \mapsto\left(\begin{array}{l}w \\ u\end{array}\right)$, for $w, u$ words in an alphabet $A$ ) are representative functions. Also, it is well known that representative functions on $\mathbb{Q}^{\mathfrak{S}}$ is an algebra under pointwise product (see [5], chapter 1 ). We recover this result in the Theorem 2 . 


\section{Acknowledgements}

The author would like to thank Christophe Reutenauer and Franco Saliola for their advice and support during the preparation of this paper, and also to the two anonymous referees for their helpful comments.

\section{References}

[1] M. Aguiar, F. Sottile. Structure of the Malvenuto-Reutenauer Hopf algebra of permutations. Adv. Math. 191 no. 2, 225-275 (2005).

[2] K. T. Chen, R. H. Fox, R. C. Lyndon. Free differential calculus, IV - The quotient groups of the lower central series, Ann. Math 65, 163-178 (1978).

[3] S. Eilenberg, S. MacLAne. On the groups $H(\Pi, n)$, Annals of Mathematics, 58, 55-106 (1953).

[4] J.-Y. Enjalbert, H. N. Minh. Combinatorial study of colored Hurwitz polyzêtas. Discrete Mathematics. 312, 3489-3497 (2012).

[5] G. P. Hochschild. Basic theory of algebraic groups and Lie algebras. Graduate Texts in Mathematics, 75. Springer-Verlag, New York-Berlin (1981).

[6] M. Hoffman. Quasi-Shuffle products. Journal of Algebraic Combinatorics. 11, 49-68 (2000).

[7] S. A. Joni, G.C. Rota. Coalgebras and bialgebras in combinatorics. Umbral calculus and Hopf algebras (Norman, Okla., 1978), pp. 1-47, Contemp. Math., 6, Amer. Math. Soc., Providence, R.I., (1982)

[8] D. Knuth. The Art of Computer Programming, Vol. 1, Fundamental Algorithms, Addison-Wesley, Reading, Massachusetts (1968).

[9] M. Lothaire. Combinatorics on words, Encyclopedia of Mathematics, Vol 17. Addison-Wesley, Reading, MA (1983).

[10] C. Malvenuto, C. Reutenauer. Duality between quasi-symmetric functions and the Solomon descent algebra. J. Algebra. 177, 3, 967-982 (1995).

[11] K. Newman, D. Radford. The cofree irreducible Hopf algebra on an algebra. Amer. J. Math. 101, no. 5, 1025-1045 (1979).

[12] P. Ochsenschläger. Binomialkoeffizzenten und Shuffle-Zahlen, Technischer Bericht, Fachbereich Informatik, T. H. Darmstadt (1981).

[13] S. Poirier, C. Reutenauer. Algèbres de Hopf de tableaux. Annales des Sciences Mathématiques du Québec 19, no. 1, 79-90 (1995).

[14] D. E. Radford. A natural ring basis for the shuffle algebra and an application to group schemes. $J$. Algebra 58, 432-454 (1979).

[15] C. Reutenauer. Free Lie algebras, The Clarendon Press Oxford University Press (1993).

[16] Y. Vargas. Hopf algebra of permutation pattern functions (2014). 